\title{
A Robust Approach in Iris Recognition for Person Authentication
}

\author{
Chinni. Jayachandra ${ }^{1}$ H. Venkateswara Reddy ${ }^{2}$ B. Suresh Kumar ${ }^{3}$ B. Sruthi ${ }^{4}$ \\ ${ }^{1}$ M.Tech (C.S.E), VCE, Hyderabad, India, \\ ${ }^{2}$ Associate Professor in CSE, VCE, Hyderabad, India, \\ ${ }^{3}$ M.Tech (C.S.E), VCE, Hyderabad, India, \\ ${ }^{4}$ M.Tech (C.S.E), VCE, Hyderabad, India,
}

\begin{abstract}
In Iris recognition authentication process, iris and sclera are used as the previous inputs using to recognize the eye with different mechanisms like segmentation combining with different versions. In this paper, entirely biometric-based personal verification and identification methods have gained much interest with an increasing accent on safety. The iris texture pattern has no links with the genetic structure of an individual and since it is generated by chaotic processes externally visible patterns imaged from a distance. Iris patterns possess a high degree of randomness and uniqueness. Here we propose two algorithms they are K-Means algorithm and canny Edge Detection. Totally eight process acting to identify the pupil and also for iris recognition. Comparing images $k$ - means algorithm is to give accurate contest. As a final point it leads to open authentication person details from database.

Key words - Median filter, Iris Radius Detection, Iris Unrolling, Iris Recognition, Pupil Detection, Canny edge detection algorithm, $K$-means algorithm.
\end{abstract}

\section{Introduction}

Recognizing individuals based on biometric systems is increasing rapidly in organizations, industries and others. It mainly focuses on security throughout the world and it is so far increasing. By examining physical or behavioral characters of human beings, biometric systems are reliable [1] which are unique among all individuals. In recognition process biometric systems are deploying and enhancing the security, reliability, convenience and efficiency. Based on uniqueness and stability of the biometrics during human's lifetime, it has been utilized as an expedient method for the recognizing process for many years. There are few biometric systems are available in world like finger prints, palm, signature, face, DNA, retina, ear and iris. Iris recognition is the process of recognizing a person by analyzing the random pattern of the iris images. The automated method of iris recognition is relatively young, existing in patent only since in 1994.The human iris, an annular region located around the pupil and covered by the cornea, can provide independent and unique information of a person. Among these iris is one of the better authentication method for verification and identification of person in both modes. Iris is a ring like chromatic texture between the black central pupil and white colored sclera in the eye. The inner and outer circular templates in eye are not in a standard circular shape.

Complex characteristics exist in iris pattern exhibit the iris as an important, convenient and noninvasive natural identification means. From past years iris is utilized as identification systems rapidly. The existing algorithms are using two circular templates to identify the eye. But they are not in a standard circle shape it leads to iris legacy and difficult to find proper identification. From past iris recognition is proposed as a reliable biometric system in 1987 by L. Form [2]. Although the correlation and the structure of the iris is genetically link, the details of the pattern are not. Iris develops during prenatal growth through a process of tight forming and folding of the tissue membrane. Genetically identical an individual's irides are unique and structurally distinct, which allows for it to be used for recognizing purposes. Iris based biometric, on the other hand, involves analyze features found in the colored ring of tissue that surrounds the pupil. Identity of verification and authentication increasing day by day to provide security for this iris recognition is more accurate which cannot change by human age.

\subsection{Relate Work}

Daugman developed Daugman's theory using 2-d Gabor filter phase quantification and the code identification system [3,4]. Wildes given a theory based on the multiscale Gaussian filters for iris identification system [5]. Boles recommended a method based on the wavelet transformation algorithm to iris recognition [6]. Boles and Boashash's [7] given that iris images are analyzed in a 1-D dyadic wavelet transform in different resolution levels, using wavelet results the feature vector of the iris image was extracted. Junzhou Huang given iris segmentation and Edge extraction is using phase congruency to identify the iris [8]. H. Proenca and L.A. 
Alexandre proposed Iris segmentation methodologies for non-cooperative recognition [9]. Chung-Chih Tsai given iris segmentation based on possibilistic fuzzy logic method to iris recognition using local vectors [10]. Li Ma, Tieniu Tan is given a method Characterizing Key Local Variations for iris recognition [11]. LiYu and David Zhang given iris recognition based on relative distance of key point [12].

C. Sanchez-Avila and R. Sanchez-Reillo used two approaches using Gabor filters and multiscale zerocrossing representation for iris recognition to make efficient [13]. Ahmad M. Sarhan gave an approach using with Discrete Cosine Transform and Artificial Neural Networks for iris pattern recognition [14]. Lenina Birgale and Manesh Kokare used Ridgelets with False Acceptance Rate (FAR) and False Rejection Rate (FRR) for iris recognition [15]. Hui Zhang proposed Rectangle Conversion for folded eye and for grouping KNN method [16]. Richard P. Wildes proposed Distinctive features of the iris are manifest across a range of spatial scales. Pattern matching is well served by a band pass decomposition spanning high to low spatial frequency [17]. Hugo Proenca developed analysis for error rate in iris segmentation process regarding accuracy [18]. Kevin W. Bowyer gave a survey on iris recognition in biometrics [19]. Makram Nabti develops an effective and fast iris recognition system using addition of multiscale feature extraction technique [20]. The past techniques like multiscale, wavelets, local features, edge extraction and segmentation are used rapidly to increase the accuracy and make simple.

\subsection{Outline:}

In eye there are inner and outer boundaries which are not in a standard circular shape. The iris is a muscle with in the eye regulates the size of the pupil, controlling the amount of light, which enters the eye. It is the colored portion of the eye with coloring based on the amount of melatonin pigment within the muscle as shown in Fig.1.In this paper, we find the edges of two circular templates and we concentrate on only pupil. It helps in if there is any iris legacy and texture loss in image. Our approach is having iris recognition system is composed of eight main stages they are Scanning, RGB to gray scale, median filters, pupil center detection, canny edge detection, iris radius, iris localization and iris unrolling. In addition to this K-means algorithm is used to compare the image and to match the image form the database. According to the matching value person is authenticated and retrieve person details.

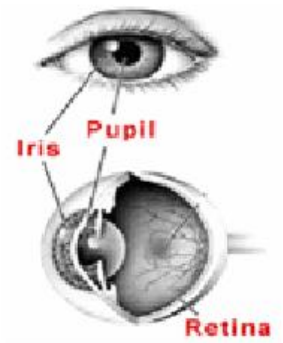

(a)

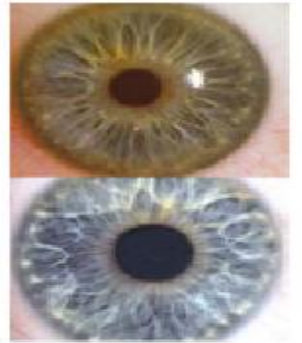

(b)

Fig 1. (a) Iris and Pupil in eye. (b) Iris and pupil Structure with colors.

In eye there are different color membrance of iris like blue, gray, red, green etc. But pupil is always having black color. In this paper mostly concentrate on pupil and next iris. The structure of pupil and iris with color is shown in above fig. 1 .

\section{An Iris Recognition System Is Composed Of Eight Stages}

Identity of verification and authentication of person is increasing rapidly for this iris recognition is the one of the best biometric system. To improve this recognition process proposed method is developed using eight stages. In this canny edge detection algorithm is mainly used to identify the edges of pupil and iris. In this recognition process we mainly focuses on pupil.

The architecture for pupil identification for iris recognition process is shown in fig. 2 with process flow. The initial step is scanning the given input image, converting RGB color image to Gray scale image, median filters to reduce the noise in images, Pupil detection by assigning center point, canny edge detection algorithm which help to identify the edges of image (pupil and iris), finding radius of iris and pupil, iris localization and finally iris unrolling as shown below. At last the result is obtained with accuracy and fine edges to without iris legacy and texture loss, which helps to match the image with database images to authenticate person information. 


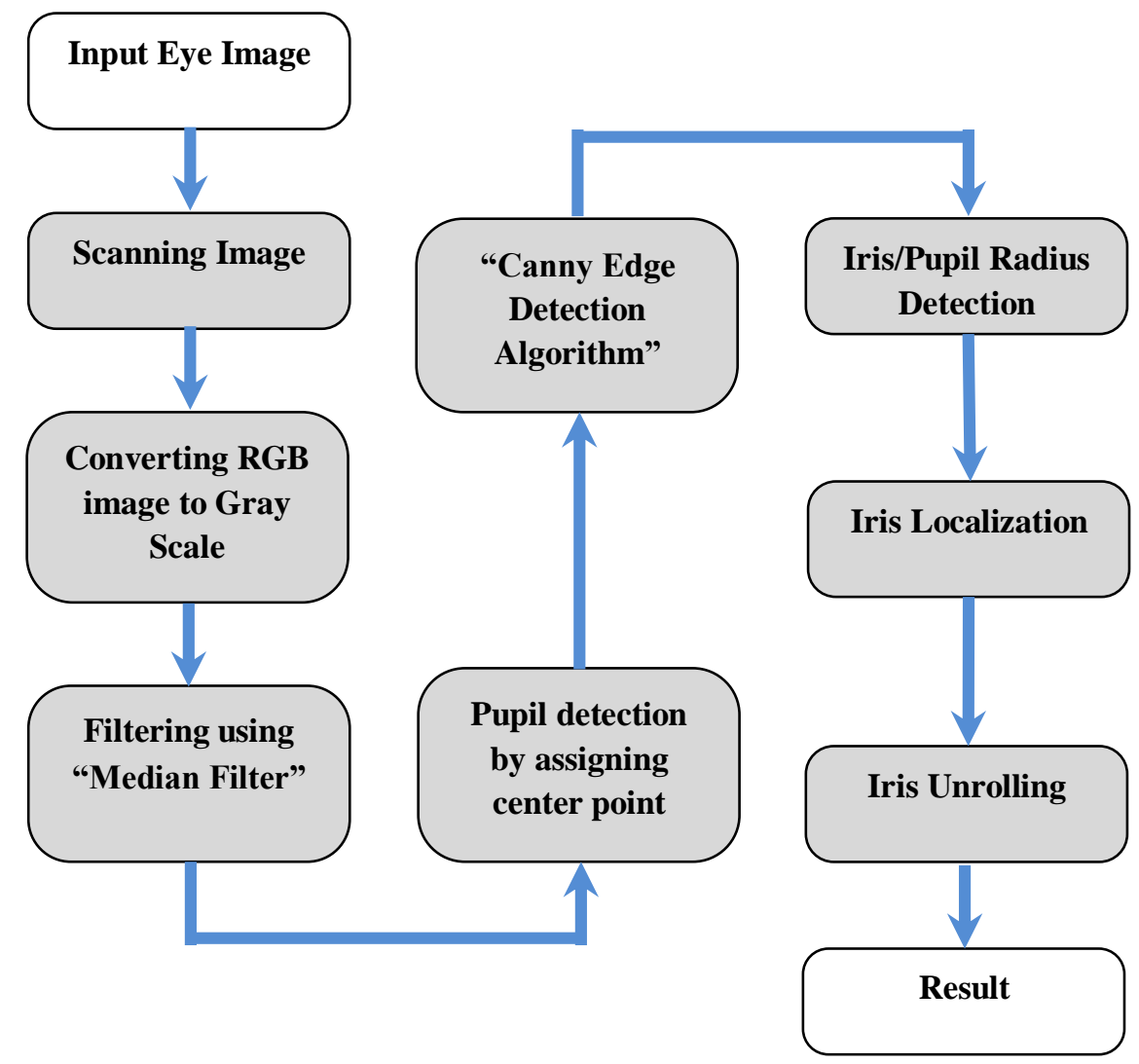

Fig 2. Architecture for Iris recognition

The Iris recognition process using pupil is follows:

\subsection{Scanning Eye}

Initially collect all eye images stored in dataset. In next process is scan process input to any one eye image. This is the process which is used to pick the image and scan the image from relevant database. In process flow it is treated as scanned input image which is used to continue next processes.

\subsection{RGB Image Converting to Gray Scale Format}

In next step taking scanned image which is having RGB color image because iris is having different colors like green, black, brown, red, blue. To find edges we need to convert the RGB to Grayscale image. The RGB encoding of pure red is $(255,0,0)$, pure green $(0,255,0)$ and pure blue is $(0,0,255)$. In all RGB encodings, the first value is the amount of red, and second value is the amount of green, and the third value is the amount of blue. Range of the 3 numbers is (0-255). Grayscale images are rendered in black, and white, and all the shades of gray in between that process. The format encoding of any gray values is a set of three equal numbers, (a, a, a), where $\mathrm{x}$ is some integer between 0 and 255 . For instance, white is $(255,255,255)$, black is $(0$, $0,0)$ and medium gray is $(127,127,127)$. The higher the numbers lighter the gray. To convert a non-neutral color to its equivalent grayscale value, next must compute a weighted average of the green, red, and blue values representation are shown in fig 3.

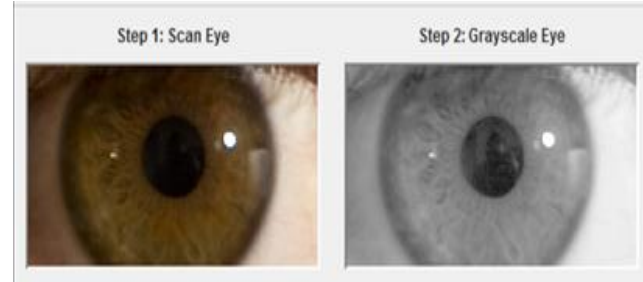

Fig 3. Converting RGB to Grayscale

\subsection{Median Filter}

In image processing, it is often desirable to be able to perform noise reduction in image or signal. Median filtering is very widely used in digital image processing because it preserves edges while removing 
noise. From the gray scale image remove the noise data like lightning, illusions, blurred data etc. This filters used before edge detection. The idea is to examine a sample of the input and decide if it is representative of the signal. This is performed using a window consisting of an odd number of samples. The values in the window are sorted into numerical order. The median value, the sample in the center of the window, is selected as the output. The oldest sample is discarded, a new sample acquired, and the calculation repeats.

\subsection{Pupil detection by assigning center point}

To perform the fourth step, find the center of the pupil to orient the coordinate system at the center of the eye. Pupil is main process to identify the iris and eye. After completing median filtering directly assign the center of eye which leads to focus on pupil as shown in fig 4.

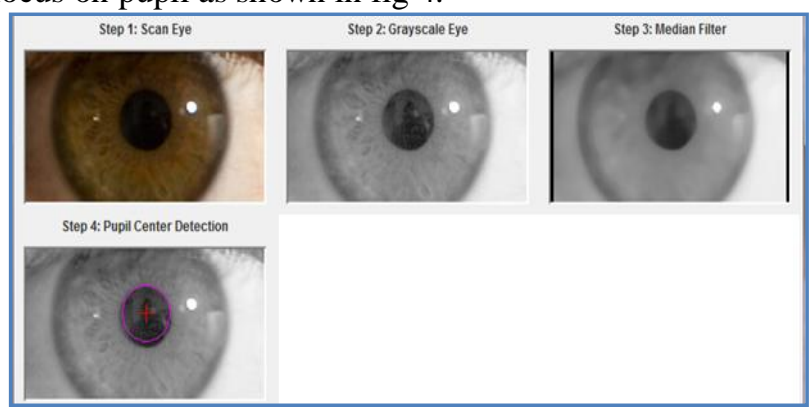

Fig 4. Pupil detection by assigning center point

\subsection{Canny Edge Detection Algorithm}

The canny edge detection algorithm is to identify the edges of image with gradient points. After identifying pupil center for that image apply this algorithm which leads to find iris and pupil boundaries. When the grayscale intensity of the image is changed to find the edges basically canny algorithm is used. This gives the effective edges of eye. This algorithm processed in following steps:

Step 1: Smoothing technique is to remove that noise is mistaken for edges, noise must be reduced and also remove the noise from the blur images.

Step 2: From the smoothed images the gradient points are determines each pixel by applying Sobel-operator. First step is to approximate the gradient in the $\mathrm{x}$ - and y-direction respectively by applying the kernels. The gradient magnitudes are also call as edge strengths, can then be determined as Euclidean distance measured by applying the law of Pythagoras as shown in Equations (1) and (2).

(1) Sometimes it is easy by applying Manhattan distance measure shown in Equation.

(2) To diminish the computational complexity.

$$
\begin{aligned}
& |\mathrm{G}|=\sqrt{\mathrm{G}_{\mathrm{x}}^{2}+\mathrm{G}_{\mathrm{y}}^{2}} \\
& |\mathrm{G}|=\left|\mathrm{G}_{\mathrm{x}}\right|+\left|\mathrm{G}_{\mathrm{y}}\right|
\end{aligned}
$$

Where, $G_{x}=$ gradients in the $x$-direction and $G_{y}$ are the gradients in the y-directions respectively. Gradient magnitude determines edges with respect to an image clearly. Though, the edges are typically broad and do not indicate precisely where the edges are. The direction of the edges must be determined and stored as shown in Equation (3).

$$
\theta=\arctan \frac{G_{y}}{G_{x}}
$$

Step 3: Non-maximum suppression is to convert the blurred edges in the image of the gradient magnitudes to make sharp edges. Basically this is done by preserving all local maxima in the gradient image, and deleting everything else. The algorithm is for each pixel in the gradient images is: (a) Round the gradient direction $\theta$ to nearest coordinate, equivalent to the use of 8-connected vicinity. (b) Contrast the edge strength of the existing pixel with the edge strength of the pixel in the optimistic and pessimistic gradient direction. (c) If the edge strength is large for current pixel, then conserve value of the edge strength and if not stem the value.

Step 4: Double thresholding mechanism is to assign edge pixels stronger than the high threshold are marked as strong, edge pixels weaker than the low threshold are concealed and edge pixels between the two thresholds are marked as weak. Because some of edges will probably be true edges in the image, but 
some may cause noise or color variations for instance due to rough surfaces. The simplest way to distinguish between these would be to use a threshold, and then only strongest edge value would be preserved.

Step 5: Edge tracking by hysteresis Edge tracking can be determined by BLOB-analysis (Binary Large Object). Using 8-connected neighborhood it connect to Blob's. Blob's should contains at least one strong edge pixel is then preserved, while other Blob's are hidden.

Canny edge detection algorithm provides:

Good detection - the algorithm should mark as many real edges in the image as possible.

Good localization - edges marked should be as close as possible to the edge in the real image.

Minimal response - a given edge in the image should only be marked once, and where possible, image noise should not create false edges.

With this step edges will obtained with pupil and iris boundaries respectively.

\subsection{Pupil and Iris Radius Detection}

Center of the iris can be computed by examining the shift vectors of the chords. Looking at both sides of a chord and comparing their lengths and width and radius can be computed. The center was shifted by this vector it would equal to two components of the chord. By doing this with two chords two $\mathrm{x}$ different offset vectors can be computed.

\subsection{Iris Localization}

This process is mostly for visual purposes and refers to removing erroneous information from the original image outside of the iris radius, whereby leaving one image within the bounds of the iris radius induction. Before eye centre localization, a pre-processing step needs to be taken. Since reflections that affect the results in a negative way, frequently appear on the eye, a reflection removal step is implemented. Such highlights are usually bright areas consisting of no more than a few pixels.

\subsection{Iris Unwrapping (Unrolling)}

The integro-differential operator proposed by Daugman[3] locates the pupil, iris inner and outer boundaries as well as the up and down eyelid boundaries.

$$
\begin{gathered}
\max _{\left(\mathrm{r}, \mathrm{x}_{0}, \mathrm{y}_{0}\right)}\left|\mathrm{G}_{\sigma}(\mathrm{r}) * \frac{\partial}{\partial r} \int_{\mathrm{r}, \mathrm{x}_{0}, \mathrm{y}_{0}} \frac{\mathrm{I}(\mathrm{x}, \mathrm{y})}{2 \pi \mathrm{r}} \mathrm{ds}\right| \\
\mathrm{G}_{\sigma}(\mathrm{r})=\frac{1}{\sqrt{2 \pi \sigma}} \mathrm{e}^{-\frac{\left(\mathrm{r}-\mathrm{r}_{0}\right)^{2}}{2 \pi^{2}}}
\end{gathered}
$$

Where $\mathrm{I}(\mathrm{x}, \mathrm{y})$ represents the eye image, are $\left(\mathrm{r}, \mathrm{x}_{0}, \mathrm{y}_{0}\right)$ parameters that correspond to a circle of radius and center coordinates $\left(\mathrm{x}_{0}, \mathrm{y}_{0}\right)$, respectively. Is a radial $\mathrm{r}$ smoothing Gaussian function with center and standard deviation? One application of this function is that it searches the entire eye image for integrations along different circular contours with center coordinates and an increasing radius. The maximum contour integral derivative found will then be classified as the most likely circle tracing the iris. In a similar manner, the circular boundaries for the pupil and iris regions are localized by search process through the entire iris image for the maximum integration along various circular contours. In addition, Daugman approximates the upper and lower eyelids with two open curves that are part of two different circles. Finally, the iris region surrounded by the upper and lower eyelids as well as the extracted circular pupil and iris boundaries are used for further feature extraction in the iris recognition process. This method, referred to as "Daugman's Rubber Sheet Model", is developed by Daugman(2003) to map the sampled iris pixels from the Cartesian coordinates to the normalized 65polar coordinates in order to accomplish a size-invariant sampling of the original iris points. After unwrapping the output of our process is as shown in below fig 5. 


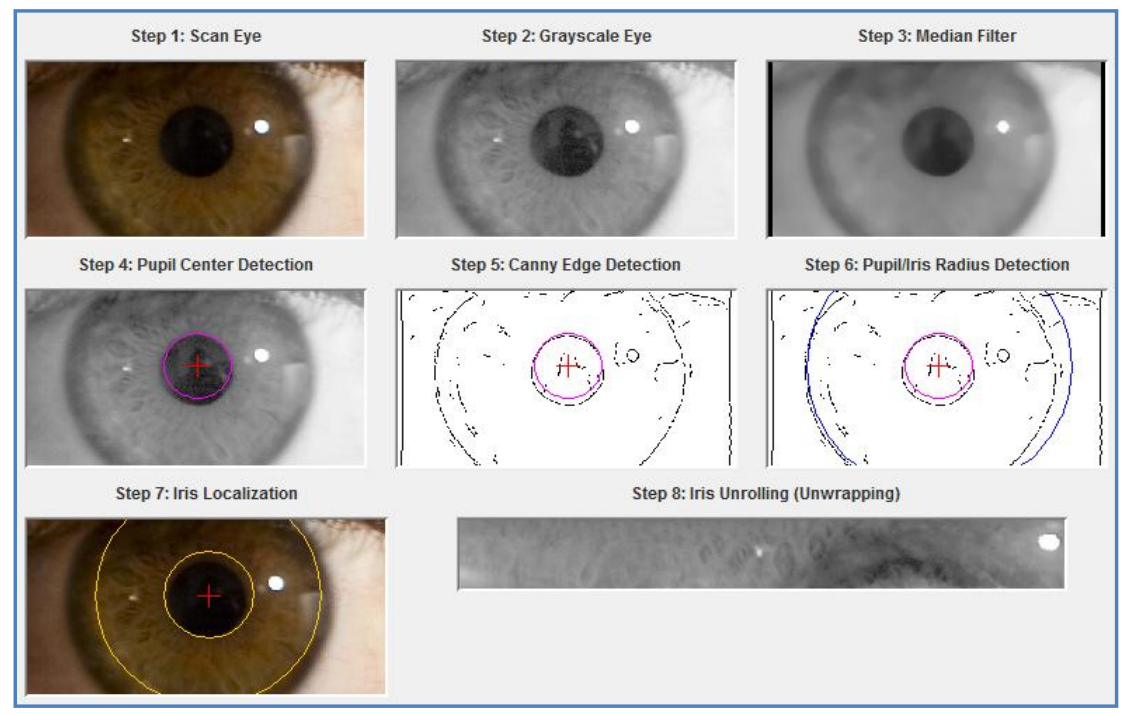

Fig 5. Iris recognition eight process output

After completion of this process pupil is identified without loss of texture and legacy. Next section, to match or compare the image and find the proper image to given input using K- Means algorithms.

\section{K-Means Algorithm for Comparison of Images}

To compare the images K-Means algorithm is reliable in our approach. Identifying pupil from the image shown in previous process. To recognize the pupil from the K-means is one of the simplest unsupervised learning algorithms that solve the well known clustering problem. The procedure follows a simple and easy way to classify a given data set through a certain number of clusters (assume k clusters) fixed a priori. The main idea is to define $\mathrm{k}$ centroids, one for each cluster. These centroids should be placed in a cunning way because of different location causes different result. So, the better choice is to place them as much as possible far away from each other. The next step is to take each point belonging to a given data set and associate it to the nearest centroid. When no point is pending, the first step is completed and an early group age is done. At this point we need to re-calculate k new centroids as gray centers of the clusters resulting from the previous step. After we have these $\mathrm{k}$ new centroids, a new binding has to be done between the same data set points and the nearest new centroid. A loop has been generated. As a result of this loop we may notice that the $\mathrm{k}$ centroids change their location step by step until no more changes are done.

$$
\mathrm{J}=\sum_{\mathrm{j}=1}^{\mathrm{k}} \sum_{\mathrm{i}=1}^{\mathrm{n}}\left\|\mathrm{x}_{\mathrm{i}}^{(\mathrm{j})}-\mathrm{c}_{\mathrm{j}}\right\|^{2}
$$

Where $\left\|x_{i}^{(j)}-c_{j}\right\|^{2}$ is a chosen distance measure between a data point $x_{i}^{(j)}$ and the cluster centre $c_{j}$, is an indicator of the distance of the $n$ data points from their respective cluster centers.

The k-means algorithm can be run multiple times to reduce this effect. Here based on the pupil, the iris identification process runs. The input image is go to the related color set in that the image edges are used to match the same edge form the nearest image edges. It continues till it finds the exact image. After matching image it open the person details to process future steps. If suppose we take net banking system for that iris recognition is first it capture the image and start matching and it opens the bank account as shown in fig 6 . 

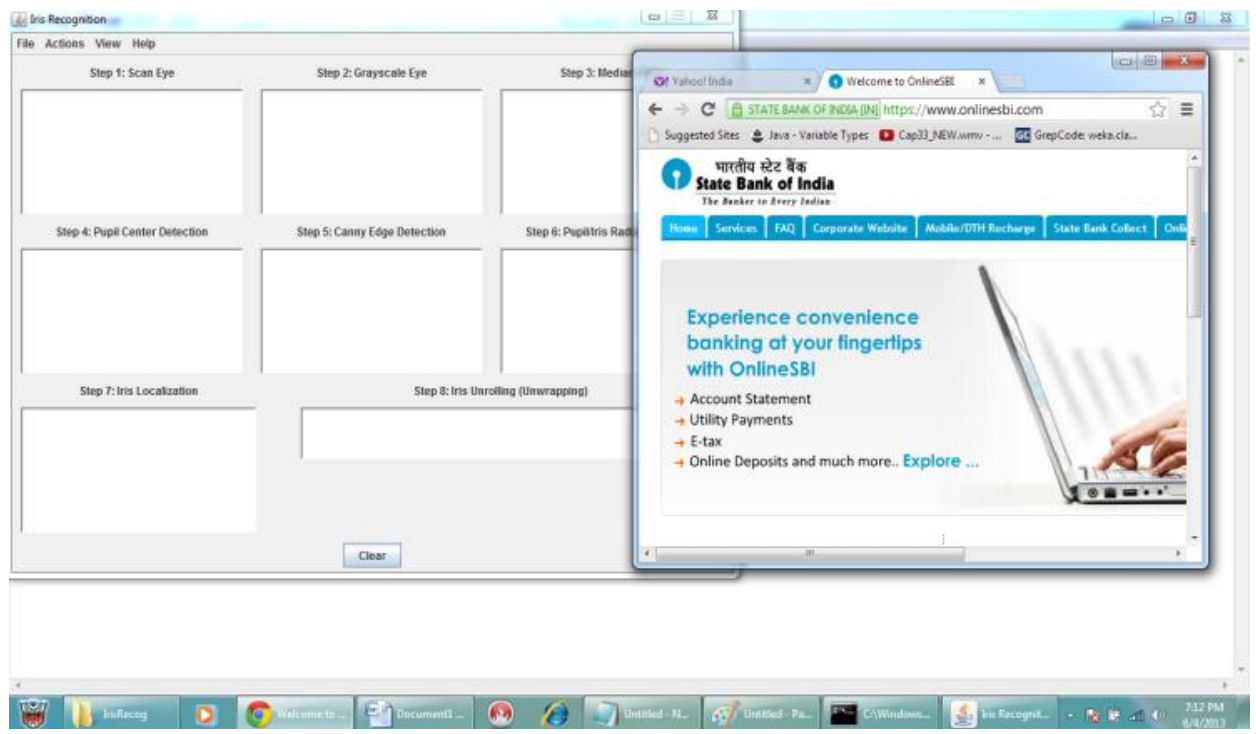

Fig 6. Representation of authentication process with bank application

\section{Performance and Experimental Results}

In previous there are many models for segmentation process but it is not applicable to find high accuracy and it is having some defects like loss of texture. Based on pupil we can find the Iris recognition with high accuracy because it doesn't have texture loss and legacy. In our approach we find the good accuracy rate compare with other models, some sample images as shown in below by using above eight processes in step by step manner:

Image 1:

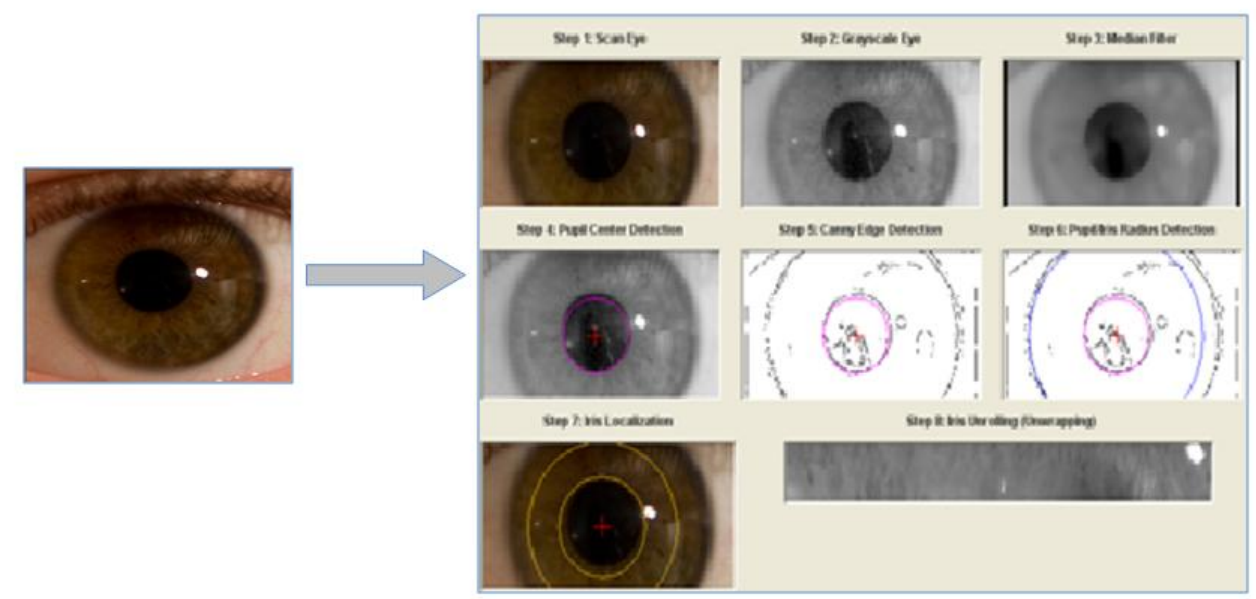

Image 2:

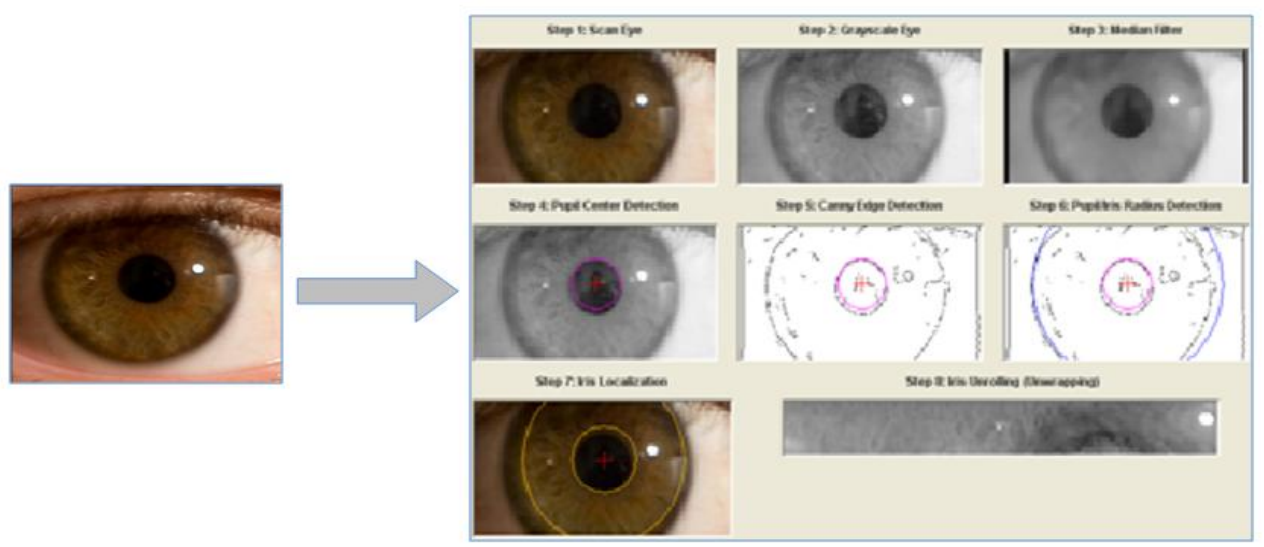


Image 3:
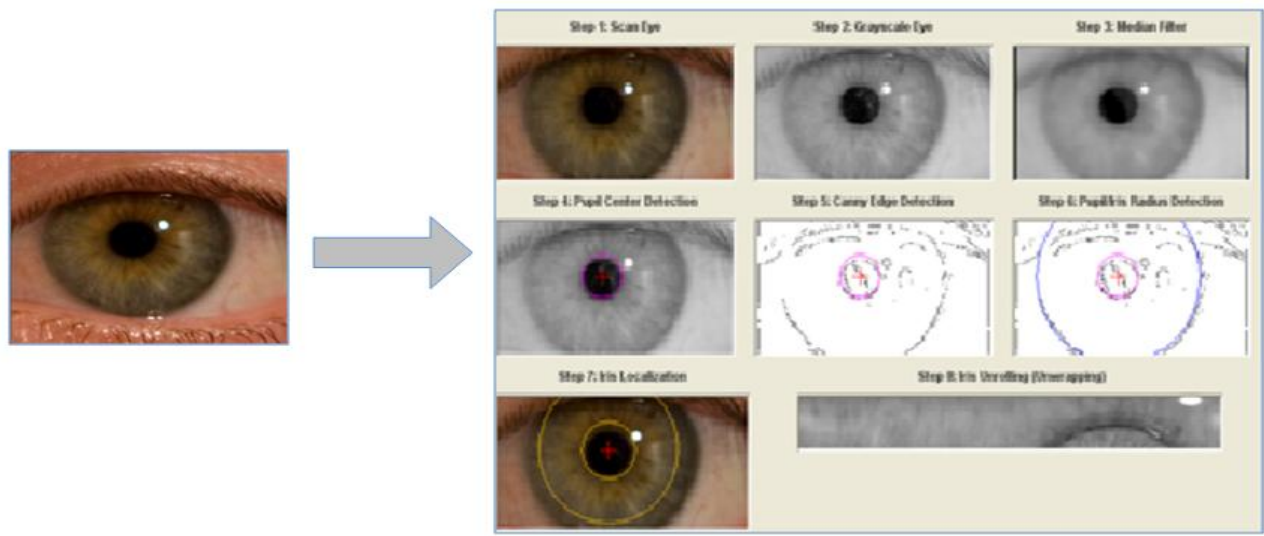

Fig 6. Iris recognition with different sample images in composed eight step process

According to existing system iris recognition is improved and accuracy rate is high compared with the existing system as shown in Fig 7. In Table 1, there the values which shows accuracy rate between existing and proposed systems.

TABLE 1

\begin{tabular}{|l|llllllll|}
\hline Existing & 1 & 1.354 & 2.721 & 3.099 & 4.0123 & 4.367 & 4.789 & 5.355 \\
\hline Proposed & 1.134 & 1.789 & 3.345 & 3.987 & 4.712 & 4.988 & 5.238 & 5.732 \\
\hline
\end{tabular}

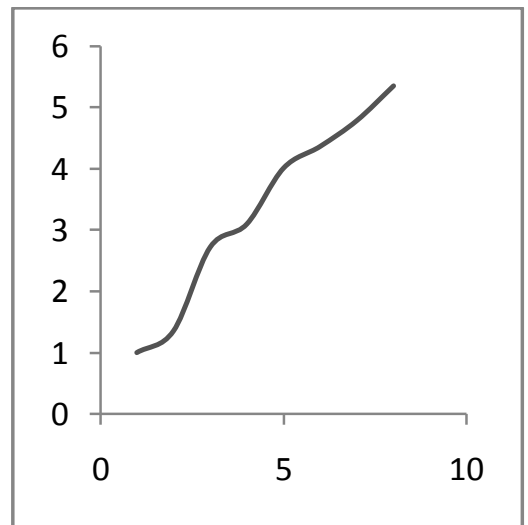

(a)

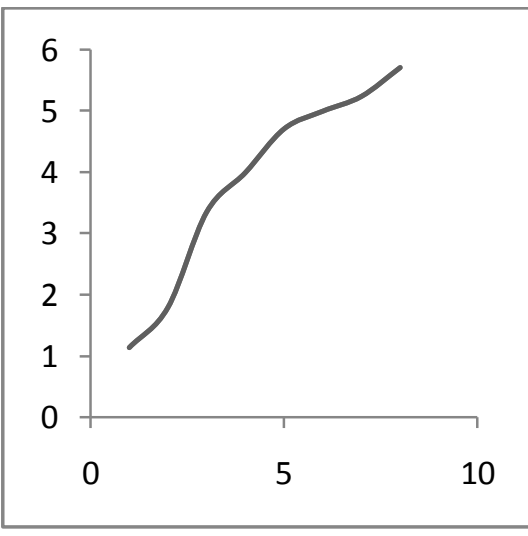

(b)

Fig 7. Graph for Performance (a) Existing and (b) Proposed system.

\section{APPLICATIONS}

The most popular biometric authentication from the last few years is Iris Recognition. It mainly focuses on entry control, ATMs and Government program applications. Recently in India to provide identity for citizen's adhar card is developed. To issue that card person need to complete personal verification in that process they used biometric systems like finger prints and eye detection. In that iris recognition used to provide identity and also to verify the person. It is applicable in organizations security to authenticate the employee with their details. Some organizations companies have realized the advantages of biometric authentication for networks and offer products to achieve this scheme. Products offered include fingerprint analysis, iris recognition, voice recognition or combinations of these. It is applicable for bank applications like ATM, a customer simply walks up to the ATM and looks in a sensor camera and if he is authenticated it allows continuing the transaction otherwise not. Iris recognition is highly accurate, easy to use and virtually fraud proof means to identify customer's details.

\section{Conclusion}

In Iris recognition process the traditional approaches methods cause the problem of pupil legacy and loss of texture. In eye, inner and outer circular templates are not in a standard circular shape. Our approach is 
based on canny edge detection and K-means algorithm effectively improves the iris positioning accuracy, through the combination of K-Means algorithm to raise the accuracy and speed of recognition. It guarantees the effective pupil detection to get accurate iris identification. In future we used recognition techniques using multichannel median filtering. Hence, they have used 8-directional median filters with multiple frequencies to capture both global and local details in an iris image. The mean and variance of these Median filtered images are used as features for the matching process. Input from dataset Eye Image next Eight Process Finishing operations and Image comparison, Iris radiation, pupil detection operation using K-means Algorithm and canny edge detection algorithm. This approach is efficient, convenient and low cost implementation.

\section{References}

[1] Jain, A.K., Flynn, P., Ross, A. (Eds.), 2007. Handbook of Biometrics. Springer-Verlag.

[2] L. Flom, A. Safir, Iris Recognition System, U.S. Patent 4641 349, 1987.

[3] J. G. Daugman, "High confidence personal identification by rapid video analysis of iris texture," in Proc. IEEE Int. Carnahan Conf. SecurityTechnol., 1992, pp. 1-11.

[4] J G Dauglllan, "High confidence visual recognition of Persons by a test of statistical independence". IEEE Transactions on Pattern Analysis and Machine Intelligence, 1993, 15(1):1148-116.

[5] R P wildes, et al. A system for automated iris recognition[C]. In Proceedings of the Second IEEE Workshop on Applications of Computer Vision. Sarasota, Horida, 1994. 121-128.

[6] W W Boles. A wavelet transform based technique for the recognition of the human iris. In Proeeedings of the 1996 International SylnPosium on Signal Processing and its Applications, Gold Cost, Australia, 1996.

[7] W. W. Boles and B. Boashash, "A human identification technique using images of the iris and wavelet transform," IEEE Trans. Signal Process. vol. 46, no. 4, pp. 1185-1188, Apr. 1998.

[8] Junzhou Huang, Yunhong Wang, Tieniu Tan, Jiali Cui, “A New Iris Segmentation Method for Recognition”, Proceedings of the 17th International Conference on Pattern Recognition, IEEE, 2004.

[9] H. Proenc, a and L.A. Alexandre, "Iris segmentation methodology for non-cooperative recognition". In IEE Proc.-Vis. Image Signal Process. Vol. 153, No. 2, April 2006.

[10] Chung-Chih Tsai, Heng-Yi Lin, Jinshiuh Taur, and Chin-Wang Tao, "Iris Recognition Using Possibilistic Fuzzy Matching on Local Features". In IEEE Transactions on Systems, Man, and Cybernetics-Part B: Cybernetics in 2010.

[11] Li Ma, Tieniu Tan, Yunhong Wang and Dexin Zhang, "Efficient Iris Recognition by Characterizing Key Local Variations". In IEEE Transactions On Image Processing, Vol. 13, No. 6, June 2004.

[12] LiYu, David Zhang," The relative distance of key point based iris recognition”. In Pattern Recognition Society, Elsevier, 2006.

[13] C. Sanchez-Avila and R. Sanchez-Reillo, "Two different approaches for iris recognition using Gabor filters and multiscale zerocrossing representation". In Pattern Recognition Society, Pergamon, Elsevier, 2004.

[14] Ahmad M. Sarhan, "Iris Recognition Using Discrete Cosine Transform and Artificial Neural Networks". Journal of Computer Science 5 (5): 369-373, 2009.

[15] Lenina Birgale and Manesh Kokare, "Iris Recognition Using Ridgelets". In Journal of Information Processing Systems, Vol.8, No.3, September 2012.

[16] Hui Zhang, "Iris Recognition Based on Grouping KNN andRectangle Conversion”. IEEE, 2012.

[17] Richard P. Wildes, "Iris Recognition: An Emerging Biometric Technology". Proceedings of the IEEE, Vol. 85, No. 9, September 1997.

[18] Hugo Proença and Luís A. Alexandre, "Iris recognition: Analysis of the error rates regarding the accuracy of the segmentation stage". Image and Vision Computing 28, pp no: 202-206, Elsevier, 2010.

[19] Kevin W. Bowyer and Karen Hollingsworth, Image understanding for iris biometrics: A survey". Computer Vision and Image Understanding 110, 281-307, Elsevier, 2008.

[20] Makram Nabti," An effective and fast iris recognition system based on a combined multiscale feature extraction technique". Pattern Recognition 41, 868-879, Elsevier, 2008. 